\title{
Analyzing the Tradeoffs Between Economies of Scale, Time-Value of Money, and Flexibility in Design Under Uncertainty: Study of Centralized vs. Decentralized Waste-to-Energy Systems
}

\author{
Michel-Alexandre Cardin ${ }^{1}$ and Junfei $\mathrm{Hu}$ \\ Department of Industrial and Systems Engineering, National University of Singapore \\ Block E1A, \#06-25, 1 Engineering Drive 2, 117576, Singapore
}

\begin{abstract}
This paper presents and applies a simulation-based methodology to assess the value of flexible decentralized engineering systems design (i.e., the ability to flexibly expand the capacity in multiple sites over time and space) under uncertainty. This work differs from others by analyzing explicitly the tradeoffs between economies of scale (EoS) - which favors designing large capacity upfront to reduce unit cost and accommodate high anticipated demand - and the time value of money - which favors deferring capacity investments to the future and deploying smaller modules to reduce unit cost. The study aims to identify the best strategies to design and deploy the capacity of complex engineered systems over time and improve their economic lifecycle performance in the face of uncertainty by exploiting the idea of flexibility. This study is illustrated using a waste-to-energy system operated in Singapore. The results show that a decentralized design with the real option to expand the capacity in different locations and times improves the expected net present value by more than $20 \%$ under the condition of economies of scale $\alpha=0.8$ and discount rate $\lambda=8 \%$, as compared to a fixed centralized design. The results also indicate that a flexible decentralized design outperforms other rigid designs under certain circumstances since it not only reduces transportation costs, but also takes advantage of flexibility, such as deferring investment and avoiding unnecessary capacity deployment. The modeling framework and results help designers and managers better compare centralized and decentralized design alternatives facing significant uncertainty. The proposed method helps them analyze the value of flexibility in small-scale urban environments, while considering explicitly the tradeoffs between economies of scale and the time-value of money.
\end{abstract}

\section{Introduction}

In design of complex engineering infrastructure systems, designers often plan for large capacity to take advantage of economies of scale $(\mathrm{EoS})$, and reduce construction and average cost per unit of capacity [1, 2]. Real estate, transportation, power generation and supply, and water management systems are examples of complex infrastructure systems often designed for the maximum expected

\footnotetext{
${ }^{1}$ Corresponding author. Phone: +65 6516 5387. Email: macardin@nus.edu.sg
} 
needs over a given planning horizon, which is typically 20-30 years. This engineering strategy may result in systems having unused capacity for a considerable amount of time, and the need for such capacity may only materialize in a distant future. This is illustrated currently by the capacity oversupply in the real estate industry in China, who is seeing slower growth after years of rapid economic development [3].

Capacity needs are affected over time by high degrees of uncertainty and change, influenced by environmental, demographic, market, regulatory, and technological forces. On the one hand, system operators may suffer significant downside on their investment if capacity needs are less than originally anticipated, because high capacity systems typically require higher capital expenditures. On the other hand, a system designed with optimal economic performance but fixed capacity may not be easily adaptable to capture more capacity demand, if needs are higher than originally anticipated. This may also result in unearned revenues, and additional opportunity costs.

Early research efforts have discussed the tradeoffs between the benefits of deferring investments and the benefits from EoS in capacity planning under deterministic conditions (e.g., [4]). In reality, however, the environment may change in the future in an unpredictable way and, therefore, uncertainties will inevitably affect the system's lifecycle performance (e.g. total profits). To account for uncertainties, techniques based on stochastic calculus and programming can be used to determine strategies for optimal capacity deployment (e.g., [5]). Uncertainties are typically modeled using a finite number of scenarios [6]. Stochastic programming models can identify the strategy with optimal economic performance by optimizing the expectation of the objective function over a range of scenarios. Such studies, however, often do no account for the ability to adapt as uncertainty unfolds.

In light of the above, there is additional value for designers, architects, researchers and planners to realize by exploiting ideas of flexibility, more specifically in terms of future capacity expansion. Flexibility is defined here as providing the "right, but not the obligation, to change the system in the face of uncertainty [7]." A flexible strategy can reduce the impact of downside uncertainties since less 
capital may be required upfront (e.g., acting like an insurance policy). In addition, it may help generating more value by positioning the system to capitalize on upside opportunities (e.g., acting like a call option on a stock - limiting downsides, improving upsides). Reducing exposure to potential losses while positioning the system to capture more profits typically improve the expected economic performance of the infrastructure system between $10 \%-30 \%$ as compared to standard design and project evaluation methods, as shown in many studies $[1,8]$. A system designed with flexibility to expand capacity as needed may provide the system operator with the ability to defer investments, and reduce net present value (NPV) of costs. Deferring capacity flexibly and modularly enables reduced expected construction costs because it avoids unnecessary capacity deployment until it is needed. It also provides the ability to add more capacity when there is higher capacity need than anticipated.

In systems design and architecture, flexibility can be implemented by starting with small capacity, and preparing the infrastructure for expansion at a later point in time. One example in the United Kingdom was to enhance the footings and columns of a multi-level car park so that additional levels could be added, as customers visits to a nearby shopping center would increase [9]. Different from strategies that expand capacity at a fixed period or for a fixed expansion size (e.g., [4]), embedding flexibility can enable designers to dynamically make decisions according to events as they unfold, thus improving the lifecycle performance of systems.

Currently, the literature focuses on analyzing the economic value of flexibility. The work done in the real options analysis (ROA) community enables quantitative evaluation of flexibility [7]. ROA helps decision-makers calculate the value of flexibility and decide whether flexibility is worthy of an additional upfront cost. This is because flexibility typically requires an additional upfront cost as a premium compared to a completely rigid infrastructure. Few studies, however, have provided systematic models to select the best strategy for deploying capacity flexibly over time and space. In addition, the time value of money and EoS are crucial factors for capacity deployment since they significantly impact the selection of the best strategy (i.e., strong EoS encourages building large M.-A. Cardin MD-15-1298 
capacity now, while a strong opportunity cost of capital or discount rate provides incentives to delay the initial investment so as to reduce NPV of costs) [4]. Similarly, few studies have discussed the tradeoffs between the time value of money and EoS in a context where uncertainty and flexibility are considered explicitly [10].

Motivated by this, this paper addresses the following research question: how can one systematically analyze and manage flexibility for capacity expansion by considering the combined effects of uncertainties, EoS, and the time value of money simultaneously? The main contribution of this work is to present a generic capacity planning model that considers the combined effects of uncertainties, EoS, and the time value of money. It extends existing methods by embedding flexibility and dynamically making decisions according to uncertain events as they unfold. The second contribution is to provide optimal solutions by solving the generic model using a simulation-based approach. The optimal results can provide guidelines for decision-makers to assess and manage flexibility for capacity expansion in operations. The third contribution relates to the application domain. Novel technology based on anaerobic digestion and gasification is currently under development. Such systems are referred as waste-to-energy (WTE) systems, and transform organic waste into electricity. It is unclear, however, how to best deploy such systems over time in urban environments. The proposed model is applied as a demonstration to the analysis of the deployment of such systems in Singapore.

The remainder of this paper is organized as follows. The related work is presented in Section 2. The capacity planning model is presented in Section 3. The case study of WTE systems is explained in Section 4. The validity of the results is discussed in Section 5. Finally, the contributions and directions for future work are summarized in Section 6.

\section{Related work}

\subsection{Flexibility in Engineering Design}

Flexibility in engineering design is a promising way to deal with uncertainty. It aims to improve the economic lifecycle performance of complex engineered infrastructure systems by dealing pro- 
actively with uncertainty, by adapting and reconfiguring the system as needed and as uncertainty unfolds. Trigeorgis [7] suggested a number of generic flexibility (or real option) strategies: 1) defer capital investment, 2) phase deployment, 3) expand capacity, 4) abandon a project, and 5) switch production inputs and/or outputs. After embedding flexible strategies in engineering designs, appraisal mechanisms are required to evaluate the flexible strategies. The value provided by the flexible strategies can be assessed quantitatively by real options analysis (ROA) (e.g., [6]). ROA methods have been adapted recently to suit the needs of an engineering context [1]. They include decision analysis, modified versions of binomial analysis, and simulation-based analysis. Cardin [8] proposed a taxonomy and a design framework organizing existing procedures to support the design of complex systems for uncertainty and flexibility, and to organize research efforts. The procedures involve five phases: (1) standard/baseline design, (2) uncertainty recognition, (3) concept generation, (4) design space exploration and (5) process management. Various design theories and methodologies have been proposed to support design activities in each phase. Thus, the taxonomy can also be used as a design framework to guide and support design activities focusing on flexibility.

Some flexible design strategies, such as expanding capacity and abandoning a project, are related to capacity deployment. For example, if demand for capacity is lower than expected, one may abandon the project to cut down on possible losses; if demand is satisfactory but not outstanding, one can maintain operations; and if capacity demand is higher than anticipated, one can expand the plant capacity to capture more opportunities for revenues and profits [11].

Motivated by the fact that flexibility can deal with uncertainty and improve the expected lifecycle performance of complex infrastructure systems, this study aims to embed flexibility in engineering designs to generate dynamic capacity expansion strategies. Dynamic capacity expansion strategies can guide decision-makers to take different actions in the subsequent stages based on actual situations. 


\subsection{Capacity Expansion Planning}

Early efforts on capacity expansion planning mainly focused on determining future expansion sizes under a deterministic environment. Manne [4] first presented an analysis for calculating the optimal size of plant capacity to be added periodically in the future. The author showed that the optimal plant capacity increases when the EoS factor decreases (more economies of scale) and decreases when the discount rate increases. Hreinsson [12] extended Manne's work to investigate the relationship between cost and capacity, and to identify how EoS affects the optimal selection for hydroelectric projects.

Recent efforts on capacity expansion planning have been devoted to extending the early studies from two perspectives. First, more decisions are now considered in the decision-making process of capacity expansion. Besides determining future expansion sizes, other decisions like expansion times and expansion locations are analyzed in the capacity expansion process. Luss [13] summarized and classified a variety of issues for capacity expansion. Since then, many studies (e.g., [14, 15]) have been carried out to address these issues. Second, techniques like stochastic programming are now used to capture uncertainties for capacity expansion (e.g., $[6,16])$. Although stochastic programming models are able to handle uncertainties for capacity expansion planning, the strategies typically exploited are not flexible. The system cannot adapt if the actual situations do not follow the modeled scenarios (i.e. solutions are typically robust, meaning that they enable the system to perform well under a wide range of variations, without the need for pro-active adjustments.)

To generate a strategy that can adapt flexibly to changing conditions, flexibility is embedded to enable capacity expansion. de Neufville et al. [9] proposed a simulation-based real options model to quantify the value of flexibility in capacity expansion for a multiple-level car park system. Mittal [17] extended Hreinsson's work [12] by real options analysis and determined that the optimal size of a design with options thinking should be smaller than that defined by Manne [4]. Most of the current studies focus on assessing the value of flexibility in capacity expansion and highlighting the value 
provided by flexibility under uncertainty [18]. Few studies, however, have analyzed how the value of flexibility is affected by considering the combined effects of EoS, the time value of money, and uncertainties [10]. Although the tradeoffs that exist between EoS and the time value of money have been analyzed in capacity expansion in a deterministic environment (e.g., [4]), there are no generic model to analyze such tradeoffs in a context where uncertainty and flexibility are considered explicitly.

This paper aims to address these issues by developing a generic model to design and manage flexibility for capacity expansion under uncertainty. The value of flexibility is investigated by considering the effects of uncertainty, EoS, and the time value of money. This work will help designers, planners, and decision-makers to systematically generate flexible strategies for capacity expansion that can be adjusted over time, and improve the expected lifecycle performance of the system.

\section{Methodology}

\subsection{Model Development}

In this section, a generic model is developed for guiding systematically the analysis of flexible capacity expansion under uncertainty. It begins by developing a deterministic capacity planning model. Subsequently, the deterministic model is extended to a stochastic model that explicitly considers uncertainty and flexibility.

Consider a planning horizon of $T$ time periods over which demand is assumed known (i.e. deterministic forecast). Let $\theta$ denote the installed capacity and $d^{t}$ denote the demand in period $t$. The objective is to determine the capacity (i.e., $\theta$ ) to satisfy demand (i.e., $d^{t}$ ) while maximizing the net present value (NPV) over the entire planning horizon. The deterministic model can be stated as follows:

$$
\begin{array}{cl}
\operatorname{Max} & N P V=-\mathcal{C}^{0}(\theta)+\sum_{t=1}^{T}\left(\frac{1}{1+\lambda}\right)^{t}\left[\mathcal{R}^{t}\left(\theta, d^{t}\right)-\mathcal{C}^{t}\left(\theta, d^{t}\right)\right] \\
\text { s.t. } & 0 \leq \theta \leq \theta_{\max } \\
& \quad d^{t} \geq 0, \quad \forall t
\end{array}
$$


Variable $\lambda$ is the discount rate (i.e. the cost of borrowing money), $\theta_{\max }$ is the upper bound of $\theta$, $\mathcal{R}^{t}\left(\theta, d^{t}\right)$ is the revenue function in period $t$, which may include for instance the tipping fee for collecting organic waste, and the revenue for selling electricity in a WTE system. $\mathcal{C}^{t}\left(\theta, d^{t}\right)$ is the cost function in period $t$. Usually, the cost function consists of the operational and maintenance cost, the land rental fee, the cost for transporting waste and the cost for disposing residues, and $\mathcal{C}^{0}(\theta)$ represents the initial investment cost. It is estimated by a power cost function that considers the EoS effects:

$$
\mathcal{C}^{0}(\theta)=K(\theta)^{\alpha}
$$

where $K$ denotes the coefficient parameter and $\alpha$ denotes the EoS factor. The lower $\alpha$ is, the stronger the economies of scale. In typical infrastructure industries, EoS range between $0.6 \leq \alpha \leq 1.0$ [2].

To extend the deterministic model to a stochastic setting, the uncertainty parameters are modeled as stochastic processes. Let $\xi=\left(\xi^{1}, \ldots, \xi^{t}\right)$ be a scenario of uncertainty, where $\xi^{t}$ is the demand in period $t$. Denote $\Omega$ as the set of all possible uncertainty scenarios. It is assumed that $\Omega$ is a finite set $\left\{\xi_{1}, \ldots, \xi_{s}\right\}$ with corresponding probabilities $p_{s} \geq 0, \sum_{s=1}^{S} p_{s}=1$. In the context of uncertainties, the model can be written as:

$$
\begin{aligned}
& \operatorname{Max} \quad E N P V=\sum_{s=1}^{S} p_{s}\left\{-\mathcal{C}^{0}(\theta)+\sum_{t=1}^{T}\left(\frac{1}{1+\lambda}\right)^{t}\left[\mathcal{R}_{S}^{t}\left(\theta, \xi_{s}^{t}\right)-\mathcal{C}_{s}^{t}\left(\theta, \xi_{s}^{t}\right)\right]\right\} \\
& \text { s.t. (2), (4) and } \\
& \xi_{s}^{t} \geq 0, \forall s, t
\end{aligned}
$$

Eq. (5) can be used to determine the optimum capacity under uncertainty. Different from Eq. (1), the revenue $\mathcal{R}_{S}^{t}\left(\theta, \xi_{s}^{t}\right)$ and the $\operatorname{cost} \mathcal{C}_{s}^{t}\left(\theta, \xi_{s}^{t}\right)$ are determined based on the decision variable $\theta$, and the uncertain factor $\xi_{s}^{t}$. To further consider flexibility, decision rules are embedded. Specifically, the decision rules are policies that guide decision-makers on how and when to expand capacity. They are 
functions of the main random variables that act as triggering mechanisms to exercise the flexibility once particular criteria are fulfilled. Standard ROA typically relies on dynamic programming and a backward induction process to assess the value of flexibility [7, 19]. As such, the decision rule approach is different, and aims to emulate the decision process more directly, making it more suitable for analysis in engineering design.

With the decision rules and the information available at each time period, decision-makers can make decisions sequentially at each period and a flexible strategy can be generated. Suppose that the decision-makers can dynamically choose a capacity level $\theta^{t}$ in period $t$ from a set of feasible capacity $\Theta^{t}$. Let $\mathrm{X}$ denote the set of all feasible capacity sequences $x$, where $x=\left(\theta^{0}, \theta^{1}, \ldots, \theta^{T}\right)$. Let $\xi^{[t]}:=\left(\xi^{1}, \ldots, \xi^{t-1}\right)$ denote the history of the uncertainty realization up to period $t$. A decision rule can be abstracted as a function $\mathcal{F}$ that maps each scenario of uncertainty $\xi$ to a capacity sequence $x$, i.e., $\mathcal{F}: \Omega \rightarrow \mathrm{X} . \mathcal{F}_{s}^{t}\left(\xi_{s}^{[t]}, \theta_{s}^{t-1}\right)$ denotes the capacity selected in period $t$ in scenario $s$, i.e., $\theta_{s}^{t}=\mathcal{F}_{s}^{t}\left(\xi_{s}^{[t]}, \theta_{s}^{t-1}\right)$. Specifically, the capacity level in period $t$ is determined based on the waste demand in period 1 to period $t-1$ and the capacity level in the last period (i.e., $\theta_{s}^{t-1}$ ). The model with uncertainty and flexibility can be written as follows:

$$
\begin{aligned}
& \operatorname{Max} \quad E N P V=\sum_{s=1}^{S} p_{s}\left\{-\mathcal{C}^{0}\left(\theta_{s}^{0}\right)+\sum_{t=1}^{T}\left(\frac{1}{1+\lambda}\right)^{t}\left[\mathcal{R}_{s}^{t}\left(\mathcal{F}_{s}^{t}\left(\xi_{s}^{[t]}, \theta_{s}^{t-1}\right), \xi_{s}^{t}\right)\right.\right. \\
& \left.\left.-\mathcal{C}_{s}^{t}\left(\mathcal{F}_{s}^{t}\left(\xi_{s}^{[t]}, \theta_{s}^{t-1}\right), \xi_{s}^{t}\right)-\mathcal{H}_{s}^{t}\left(\mathcal{F}_{s}^{t}\left(\xi_{s}^{[t]}, \theta_{s}^{t-1}\right), \theta_{s}^{t-1}\right)\right]\right\} \\
& \text { s.t. } \quad(4),(6) \text { and } \\
& \theta_{s}^{t} \in \Theta^{t} \quad \forall t, s \\
& \mathcal{F}_{s}^{t}\left(\xi_{s}^{[t]}, \theta_{s}^{t-1}\right)=\theta_{s}^{t} \quad \forall s, t=1,2, \cdots T
\end{aligned}
$$

Revenue and cost are determined based on the uncertain factors (i.e., $\xi_{s}^{t}$ ) as well as the capacity level selected in period $t$ (i.e., $\left.\mathcal{F}_{s}^{t}\left(\xi_{s}^{[t]}, \theta_{s}^{t-1}\right)\right)$. Function $\mathcal{H}_{s}^{t}\left(\mathcal{F}_{s}^{t}\left(\xi_{s}^{[t]}, \theta_{s}^{t-1}\right), \theta_{s}^{t-1}\right)$ is a cost function that calculates the costs associated with the capacity expansion in period $t$. It is determined using Eq. (10): 


$$
\mathcal{H}_{s}^{t}\left(\mathcal{F}_{s}^{t}\left(\xi_{s}^{[t]}, \theta_{s}^{t-1}\right), \theta_{s}^{t-1}\right)=\left\{\begin{array}{cc}
K\left(\theta_{s}^{t}-\theta_{s}^{t-1}\right)^{\alpha} & \text { if } \theta_{s}^{t}>\theta_{s}^{t-1} \\
0 & \text { otherwise }
\end{array}, \forall s, t=1, \ldots T\right.
$$

Eq. (10) shows that the cost associated with the capacity expansion is also calculated based on the power cost function. The capacity expansion cost only occurs when additional modules are added in the particular period.

\subsection{Model Analysis}

Once the capacity expansion model with uncertainty and flexibility is developed, the optimum capacity $\theta^{*}$ and the optimum variables for decision rule function $\mathcal{F}^{*}$ can be obtained. An example of function $\mathcal{F}$ that relates to capacity expansion can be represented as follows: expand the capacity by $\gamma \theta_{U}$ if the observed amount of waste collected in the last year is more than a certain threshold (i.e., $\left.\theta_{S}^{t-1}-\beta \theta_{U}\right)$. Here, $\theta_{U}$ is the unit capacity of an expansion module. $\gamma$ and $\beta$ are decision variables for the function $\mathcal{F} . \gamma$ represents the scale of each expansion and $\beta$ represents the security level of the current capacity; a higher $\beta$ means decision-makers are keener to expand the capacity to prevent capacity shortage.

This paper relies on a Monte Carlo simulation based algorithm to generate the stochastic scenarios, find the optimum values for decision variables (e.g., variables $\gamma$ and $\beta$ ), and quantify the value of flexibility. Three major steps are involved in the valuation: 1) generating sample scenarios (i.e., using historical data calibrated into stochastic processes); 2) exploring the design space using enumeration techniques, and; 3) calculating the value of flexibility (VOF). Eq. (5-6) calculate the ENPV for a fixed design under uncertainty, which serves as a benchmark design. Eq. (7-10) calculate the ENPV for a design with capacity expansion flexibility. The difference between the ENPVs is the VOF, which indicates the benefits of flexibly expanding the capacity under uncertainty, as compared to a rigid optimal capacity benchmark (i.e. the next best design alternative). A positive VOF shows an 
increasing ENPV produced by such capacity expansion. Generally, decision-makers should not be willing to pay more than the VOF to enable flexibility in a system design or architecture.

$$
V O F=E N P V_{\text {Flexible }}-E N P V_{\text {Benchmark }}
$$

\section{Case Study}

This section uses a Singapore WTE system as an example to demonstrate how the method described in Section 3 can be used to analyze flexible capacity expansion strategies. Two designs are analyzed in this study for deploying the anaerobic digestion (AD) technology in Singapore. The first design is a centralized design that deploys a large-scale AD plant in the western area of Singapore. ${ }^{2}$ Food waste is collected by the public waste collector (PWC) contractors within the waste collection sectors (Singapore is divided into 6 sectors under the Public Waste Collection scheme[20]). All of the collected waste is then transported to the centralized AD plant from the different sectors. The second design is a decentralized design that builds one AD plant in each waste collection sector (i.e., 6 smallscale plants are developed). The PWC contractors can then transport the collected waste to the nearby AD plant, rather than the west side of the country.

Each design has its own advantages: the main advantage for the centralized design is to reduce the average cost per unit and gain benefits from EoS, while ostensible advantages for the decentralized design are the decrease in total transportation costs, and the ability to deploy capacity modularly over time and space. Further analysis is needed to better understand the costs and benefit tradeoffs between these two designs. Flexibility for capacity expansion is embedded in both. The value of flexibility is analyzed by considering the tradeoff between EoS and the time value of money. The proposed method helps identify the best strategies for deploying new WTE systems in Singapore in the future.

\footnotetext{
${ }^{2}$ See "After Consolidation" map at http://www.nea.gov.sg/energy-waste/waste-management/a-new-uniform-fee-for-wastecollection
} 


\subsection{Model Development under Deterministic Conditions}

In this subsection, a capacity planning model is developed and evaluated under deterministic conditions. It determines the optimum capacities for both the centralized and decentralized designs to satisfy a deterministic waste demand projection. The capacities are invested in the initial phase without any changes in the future. To clarify the problem, designs without changes in the future are referred to as "fixed centralized/decentralized designs" in this paper.

The capacity planning model for the centralized design is developed by identifying the main revenues and costs of $\mathrm{AD}$ plants. The annual revenue generated by $\mathrm{AD}$ plants at year $t\left(A R_{t}\right.$ consists of two parts: the tipping fee for collecting food waste $R_{D}^{t}$ and the selling revenue for recovered electricity $\left.R_{E}^{t}\right)$. It can be calculated as follows:

$$
\begin{aligned}
& A R_{t}=R_{D}^{t}+R_{E}^{t} \\
& R_{D}^{t}=d^{t} P_{t o} \\
& R_{E}^{t}=\min \left(d^{t}, \theta\right) E_{g} P_{e} \\
& d^{t}=\sum_{i=1}^{6} d_{i}^{t}
\end{aligned}
$$

where $d_{i}^{t}$ is the recycled food waste from sector $i$ at year $t, d^{t}$ is the total recycled food waste at year $t$, $\theta$ is the capacity developed in the main site (i.e., the western regions of Singapore), $P_{t o}$ is the unit tipping fee for food waste, $E_{g}$ is the electricity generation rate, and $P_{e}$ is the unit selling price for electricity.

The annual cost at year $t\left(A C_{t}\right)$ includes four parts: the transportation cost $C_{\text {Tran }}^{t}$, the disposal cost for residue $C_{D}^{t}$, the land $\operatorname{cost} C_{L}^{t}$, and the regular operational and maintenance $(\mathrm{O} \& \mathrm{M}) \operatorname{cost} C_{O M}^{t}$. It is shown in Eq. (16):

$$
A C_{t}=C_{\text {Tran }}^{t}+C_{D}^{t}+C_{L}^{t}+C_{O M}^{t}
$$


For the centralized design, the transportation cost $C_{\text {Tran }}^{t}$ includes the fuel consumption for collecting waste within each waste collection sector as well as the fuel consumption for transporting waste from each sector to the main site.

$$
C_{\text {Tran }}^{t}=C_{\text {fuel }} \sum_{i=1}^{6}\left[\frac{d_{i}^{t}}{\operatorname{Cap}_{v}}\right]\left(D_{c o_{i}}+D_{T r_{i}}\right)
$$

where $C a p_{v}$ is the capacity for a transportation vehicle and $d_{i}^{t} / \operatorname{Cap}_{v}$ represents the total number of trips required to transport waste to sector $i . D_{c o_{i}}$ is the distance for collecting waste within sector $i$ and $D_{T r_{i}}$ is the distance for transporting waste from sector $i$ to the main site. $D_{c o_{i}}+D_{T r_{i}}$ is the total distance of one trip for sector $i . C_{\text {fuel }}$ is the unit cost for fuel consumption $(\mathrm{S} \$ / \mathrm{km})$.

The disposal cost $C_{D}^{t}$ is not only related to the quantity of impurities and residues, but is also affected by the unprocessed waste due to capacity shortage at year $t$. The reason for taking into account the unprocessed waste is that government agencies in Singapore are required to treat all food waste in $\mathrm{AD}$ plants. The waste that cannot be treated due to capacity shortage will be disposed at incineration plants with higher disposal costs. Such cost is similar to a penalty cost, which penalizes the AD designs that cannot satisfy the demand requirement. The calculation is shown in Eq. (18):

$$
C_{D}^{t}=\left(\min \left(d^{t}, \theta\right)(1-\omega+\sigma)+\max \left(d^{t}-\theta, 0\right)\right) C_{d i s}
$$

where $\omega$ is the purity rate for the recycled food waste and $\sigma$ is the residue rate. The first item of Eq. (18) calculates the quantity of impurities and residues at year $t$, while the second item calculates the quantity of waste that cannot be disposed at $\mathrm{AD}$ plants due to capacity shortage. All of this waste should be sent to the incineration plants for further disposal, and $C_{d i s}$ is the unit cost for disposing waste in incineration plants.

The land $\operatorname{cost} C_{L}^{t}$ is calculated based on the installed capacity $\theta$. It is estimated using Eq. (19):

$$
C_{L}^{t}=\rho \theta
$$

where $\rho$ is the unit land rental fee for installed capacity. The operational and maintenance $\operatorname{cost} C_{O M}^{t}$ is assumed to be a proportion of the initial cost and is calculated using Eq. (20): 


$$
C_{O M}^{t}=\pi \mathcal{C}^{0}(\theta)=\pi\left[K(\theta)^{\alpha}\right]
$$

where $\pi$ is the coefficient parameter and assumed to be $15 \%$ in this case [21].

The capacity planning model for the fixed centralized design is formulated according to Eq. (1):

$$
\begin{gathered}
\operatorname{Max} \quad N P V=-\mathcal{C}^{0}(\theta)+\sum_{t=1}^{T} \frac{A R_{t}-A C_{t}}{(1+\lambda)^{t}} \\
\text { s.t. }(12)-(20)
\end{gathered}
$$

Compared to the fixed centralized design, six small-scale plants are developed in the decentralized design, rather than one large-scale plant. The six plants are assumed to have the same capacity. In addition, the total capacity of the decentralized design is the same as the capacity of the centralized design $\theta$, as shown in Eq. (22):

$$
\theta_{i}=\theta / 6 \quad i=1,2, \cdots, 6
$$

where $\theta_{i}$ is the capacity developed in sector $i$. Since the waste in the decentralized design can be treated in the nearby AD plant within the waste collection sector, $D_{T r_{i}}$ is assumed to be 0 in the decentralized design and the transportation cost is calculated using Eq. (23):

$$
C_{\text {Tran }}^{t}=C_{\text {fuel }} \sum_{i=1}^{6} \frac{d_{i}^{t}}{\operatorname{Capv}_{v}} D_{c o_{i}}
$$

Therefore, the capacity planning model for the decentralized design is formulated as follows:

$$
\begin{gathered}
\operatorname{Max} \quad N P V=-\sum_{i=1}^{6} \mathcal{C}^{0}\left(\theta_{i}\right)+\sum_{t=1}^{T} \sum_{i=1}^{6} \frac{A R_{t, i}-A C_{t, i}}{(1+\lambda)^{t}} \\
\text { s.t. (12) - (16), (18) - (20), (22) - (23) }
\end{gathered}
$$

To gather real data to solve the above capacity planning model, a comprehensive review was done to include recent efforts in the design of $\mathrm{AD}$ plants, the technical details of $\mathrm{AD}$ plants, and commercialized applications in Europe and Singapore. Keywords such as "anaerobic digestion" and "waste to energy" - or different combinations thereof - were used in e-index search engines like Web of Science and Google Scholar. In addition to the literature survey, communications with experts in the 
of the assumptions for the capacity models.

TABLE 1 LIST OF ASSUMPTIONS FOR MODEL DEVELOPMENT

\begin{tabular}{|c|c|c|c|}
\hline Parameters & Definition & Assumptions & Comments and Source \\
\hline$C_{\text {dis }}$ & unit cost for disposing residues & S\$77/ton & $\begin{array}{l}\text { This is the dispose cost which should be paid by AD } \\
\text { plants to dispose of residues in incineration plants [20]. }\end{array}$ \\
\hline $\operatorname{Cap}_{v}$ & $\begin{array}{l}\text { vehicle capacity for collecting } \\
\text { wastes per trip }\end{array}$ & 25 tonnes & \\
\hline$C_{\text {fuel }}$ & unit cost for fuel consumption & $\mathrm{S} \$ 0.4 / \mathrm{km}$ & $\begin{array}{l}\text { The price of diesel fuel is } S \$ 1.625 \text { in Singapore [22]. It } \\
\text { is assumed one liter of diesel fuel is able to last for } 4 \mathrm{~km} \\
\text { of travel distance. }\end{array}$ \\
\hline$D_{c o}$ & $\begin{array}{l}\text { distance for collecting wastes } \\
\text { within sector } i\end{array}$ & $54 \mathrm{~km}$ & $\begin{array}{l}\text { It is assumed that the distances for collecting waste } \\
\text { within the } 6 \text { sectors are the same. }\end{array}$ \\
\hline$D_{T r_{i}}$ & $\begin{array}{l}\text { distance for transporting wastes } \\
\text { from sector } i \text { to the main sector }\end{array}$ & $\begin{array}{l}0 \mathrm{~km}, 20 \mathrm{~km} \\
25 \mathrm{~km}, 29 \mathrm{~km} \\
36 \mathrm{~km}, 40 \mathrm{~km}\end{array}$ & $\begin{array}{l}0 \mathrm{~km} \text { represents the transporting distance of the main } \\
\text { site. It is assumed that no additional effort is required to } \\
\text { transporting the wastes to the main site if the wastes are } \\
\text { collected within the main site. The rest numbers are } \\
\text { represented as the distances from other sectors to the } \\
\text { main site and are assumed based on Google map. }\end{array}$ \\
\hline$d^{t}\left(d_{i}^{t}\right)$ & $\begin{array}{l}\text { amount of recycled food wastes } \\
\text { at year } t \text { (in sector } i \text { ) }\end{array}$ & $\begin{array}{l}274 \text { tpd } \\
\text { (ton per day) }\end{array}$ & $\begin{array}{l}\text { The total recycled food waste in Singapore in } 2013 \text { was } \\
100,000 \text { tonnes [23]. }\end{array}$ \\
\hline$E_{g}$ & electricity generation rate & $230 \mathrm{kWh} /$ ton & $\begin{array}{l}\text { It is assumed that the biogas generation rate is } 150 \mathrm{~m}^{3} / \mathrm{t} \\
\text { [24].The electricity conversion rate is } 35 \% \text { and only } \\
80 \% \text { of the generated electricity can be sold to power } \\
\text { grid [21]. }\end{array}$ \\
\hline$P_{\text {to }}$ & unit tipping fee for food wastes & $\mathrm{S} \$ 65 /$ ton & $\begin{array}{l}\text { It is assumed to be slightly lower than } C_{d i s} \text { to } \\
\text { encourage organic waste separation. }\end{array}$ \\
\hline$P_{e}$ & unit selling price for electricity & $\mathrm{S} \$ 0.27 / \mathrm{kwh}$ & $\begin{array}{l}\text { It is estimated based on the Singapore electricity tariff in } \\
2013 \text { [25]. }\end{array}$ \\
\hline$K$ & & & Both coefficient $K$ and $E o S$ factor \\
\hline K & Coefficient for cost function & 305,288 & $\begin{array}{l}\alpha \text { are estimated based on the real data from [21]. } \\
\text { Detailed analysis can be found in Hu and Cardin [26]. A }\end{array}$ \\
\hline$\alpha$ & economies of scale factor & 0.8 & reasonable range of economies of scale factor is 0.4 to 1 . \\
\hline$\rho$ & $\begin{array}{l}\text { unit land rental fee for the } \\
\text { installed capacity }\end{array}$ & $\mathrm{S} \$ 816 / \mathrm{tpd}$ & \\
\hline$\sigma$ & residues rate for food wastes & $5 \%$ & $\begin{array}{l}\text { The residues rate for incineration technology is } 10 \% \text { in } \\
\text { Singapore [27].The resides rate for AD is assumed to be } \\
\text { less than } 10 \% \text { since it has high efficiency [28]. }\end{array}$ \\
\hline$\omega$ & purity rate for food wastes & $70 \%$ & $\begin{array}{l}\text { In Singapore, the food wastes from industrial and } \\
\text { commercial areas have about } 30 \%-40 \% \text { impurities [29]. }\end{array}$ \\
\hline$T$ & lifecycle period & 15 & Long-term lifecycle period \\
\hline$\lambda$ & discount rate & $8 \%$ & A general discounted rate \\
\hline
\end{tabular}

Based on these assumptions and models, the fixed centralized and decentralized designs are evaluated under a deterministic forecast (historical data for the recycled food waste in Singapore is originally from a National Environment Agency (NEA) annual report [23]). Fig. 1 shows the results of 
the fixed centralized/decentralized design analyses under the deterministic forecast. It compares the NPVs for different sizes of plants that have various EoS factors. It clearly shows that the fixed centralized design performs better than the fixed decentralized design (i.e., has a higher NPV) when taking into account EoS with $\alpha=0.6,0.7,0.8$ and 0.9 . This is mainly because the benefit from EoS in

the fixed centralized design exceeds the benefit from reducing transportation costs in the fixed decentralized design (i.e., $D_{T r_{i}}=0$ in the fixed decentralized design). Since Singapore is a small citystate, the transportation cost is not a significant portion of the total cost in this context. Therefore, reducing transportation costs by using a modular decentralized approach cannot counteract the benefits from existing EoS.

Fig. 1 NPV (expressed in S\$ million) of fixed designs capacities (expressed in tpd) under deterministic analysis

Fig. 1 also shows that the stronger the EoS (i.e., lower EoS factors), the larger the fixed design should be (e.g., $\alpha=0.6$, the optimal centralized design is 1300 tpd; $\alpha=0.7$, the optimal centralized design is 1000tpd). This is because with strong EoS, it is worth building large capacity upfront to benefit from the reduced average cost per unit capacity. The NPVs for both centralized and decentralized designs are negative values when $\mathrm{EoS}$ is characterized by $\alpha=0.9$. This is because with weaker EoS (i.e., larger EoS factor), capacity deployed upfront is more costly in present value terms, and there is no phasing or modular flexibility exploited to reduce such cost.

\subsection{Model Development under Uncertainty}

In this section, the fixed centralized/decentralized designs are evaluated under uncertainty analysis. Many uncertainties can affect the economic performance of AD systems, such as waste quantity, waste composition, waste quality, energy price and material cost. Since this paper focuses on flexibility in capacity expansion, only one factor that is affecting mostly capacity expansion (e.g., recycled food waste quantity) is modeled. A wide range of scenarios for the recycled food waste is generated. The 
performances of the optimum fixed designs are evaluated under different scenarios. Here, the recycled food waste is modeled using standard geometric Brownian motion (GBM), which can represent an overall increasing trend with some random shocks [26]:

$$
\begin{array}{rlrl}
d \xi_{i}^{t} & =\mu \xi_{i}^{t} d t+\Sigma \xi_{i}^{t} d W_{t} & t & =1,2, \cdots T, i=1,2, \cdots 6 \\
\xi^{t} & =\sum_{i=1}^{6} \xi_{i}^{t} & t & =1,2, \cdots T, i=1,2, \cdots 6
\end{array}
$$

Variable $\xi_{i}^{t}$ is the recycled food waste in sector $i$ at year $t$ and $\xi^{t}$ is the total recycled food waste at year t. The parameter $\mu$ represents an increasing trend in the recycled food waste. It is assumed to be the deterministic growth rate of food waste in Singapore (i.e., $\mu=g_{f}=12.3 \%$ ). $\Sigma$ represents the volatility. It is approximated using the standard error $\Sigma=16.3 \%$, which is obtained from regression analysis of historical waste generation data [23]. $\xi^{0}$ is the total recycled food waste at year 2013 in Singapore (i.e., $\left.\xi^{0}=274 \mathrm{tpd}\right)$ and $\xi_{i}^{0}=\xi^{0} / 6 . d t$ is a time increment of one period, which is assumed to be one year. The random variable $d W_{t}$ captures the standard Wiener variable, modeling the stochastic error at time $t$, sampled from a standard normal distribution. Based on these assumptions, 2,000 different 15-year scenarios consisting of the recycled food waste in each year for are sampled. The sample size was chosen to balance the need to provide good statistical support, while not requiring an excessive amount of computations. Fig. 2 (a) shows the recycled food waste in each sector under one stochastic scenario. Fig. 2 (b) shows five of the 2,000 runs of the total recycled food waste as well as the deterministic projection in the deterministic analysis.

Fig. 2 (a) Recycled food waste in each sector; (b) GBM simulation of total recycled food waste (5 out of 2,000 runs)

To consider uncertainty scenarios, the capacity planning model for the centralized design can be formulated according to Eq. (27):

$$
\text { Max } \quad E N P V=\sum_{s=1}^{2000} p_{s}\left(-\mathcal{C}^{0}(\theta)+\sum_{t=1}^{T} \frac{A R_{t, s}\left(\theta, \xi_{s}^{t}\right)-A C_{t, s}\left(\theta, \xi_{s}^{t}\right)}{(1+\lambda)^{t}}\right)
$$

s.t. $(12)-(20)$, and (25)-(26) 
where $\xi_{s}^{t}$ is the total recycled wastes in scenario $s$ at year $t . p_{s}$ is the probability of scenario $s$. In this evaluation, all scenarios are considered equally likely, so $p_{s}=1 / 2,000$ for all scenarios. The capacity planning model for the decentralized design can be formulated as follows:

$$
\begin{gathered}
\operatorname{Max} \quad E N P V=\sum_{s=1}^{2000} p_{s}\left(-\sum_{i=1}^{6} \mathcal{C}^{0}\left(\theta_{i}\right)+\sum_{t=1}^{T} \sum_{i=1}^{6} \frac{A R_{t, i, s}\left(\theta_{i}, \xi_{i s}^{t}\right)-A C_{t, i, s}\left(\theta_{i}, \xi_{i s}^{t}\right)}{(1+\lambda)^{t}}\right. \\
\text { s.t. (12) }-(16),(18)-(20),(22)-(23) \text { and }(25)
\end{gathered}
$$

where $\xi_{i s}^{t}$ is the recycled waste in sector $i$ in scenario $s$ at year $t$. The ENPVs for both centralized and decentralized designs are maximized with respect to $\theta\left(\theta_{i}\right)$.

The optimum fixed designs with different EoS factors are compared in the deterministic and uncertainty analyses. Results show that the optimized fixed centralized design outperforms the optimized fixed decentralized design under uncertainty analysis if the effect of EoS is taken into account (e.g., $\alpha=0.8, E N P V_{\text {cen }}=\mathrm{S} \$ 22$ million $>E N P V_{\text {decen }}=\mathrm{S} \$-5$ million). This is similar to the results of the deterministic analysis (e.g., $\alpha=0.8, N P V_{\text {cen }}=\mathrm{S} \$ 24$ million $>N P V_{\text {decen }}=\mathrm{S} \$ 6$ million). Since the fixed centralized designs generate higher economic performance than the fixed decentralized designs in both the deterministic analysis and the uncertainty analysis, the fixed centralized design with capacity 600tpd is selected as the benchmark design in this study. In addition, the result demonstrates that the ENPV of the fixed design under uncertainty $\left(E N P V_{c e n}=\mathrm{S} \$ 22\right.$ million $)$ is smaller than the NPV generated by deterministic analysis $\left(N P V_{c e n}=\mathrm{S} \$ 24\right.$ million $)$. This is caused by capacity constraints, preventing the profits from high waste generation scenarios from counterbalancing the lower profits (and losses) generated by low waste generation scenarios.

\subsection{Flexible Capacity Expansion}

In this subsection, flexibility is introduced and embedded in the system designs. Suitable real option strategies (i.e., abandonment, switching input/output, and expansion) and design enablers (i.e., mechanisms to enable flexibility in design) can be explored through flexible systems design concept generation activities. The works by $\mathrm{Hu}$ and Cardin [26], Mikaelian et al. [30] and Cardin et al.[31] are 
examples of how to do this more systematically. Here, only a capacity expansion real option is analyzed. Two flexible designs are generated: a flexible centralized design and a flexible decentralized design.

\section{1) Flexible Centralized Design}

The flexible centralized design starts with a small initial module and expands capacity (e.g., adding more digestion tanks) as desired. This design reduces exposure to losses because less capital is needed in the early stages. In addition, it helps system operators capitalize on the opportunity to receive and dispose of more feedstock by adding more capacity in the future. The flexible centralized design, however, can only expand its capacity at the main site. To enable this flexibility, more land is reserved so that $\mathrm{AD}$ plants can expand their capacity accordingly to process more feedstock. Therefore, additional costs are required to enable the flexibility. In this case study, it is assumed that the cost of enabling flexibility $C_{f}=20 \%$ of the initial investment cost as done by Lin [32].

After embedding the flexibility, decision-makers need to know when to expand and how many modules should be added, with the goal of achieving maximum economic performance. To answer these questions, decision rules should be embedded in capacity planning models. For example, this case embeds the following decision rule: IF the difference between the available food waste and the total capacity in the last year is higher than a threshold value THEN add modular designs until it reaches the upper bound (i.e., the capacity of the benchmark design), ELSE do nothing. This decision rule can be further explained using the following pseudo-code procedure:

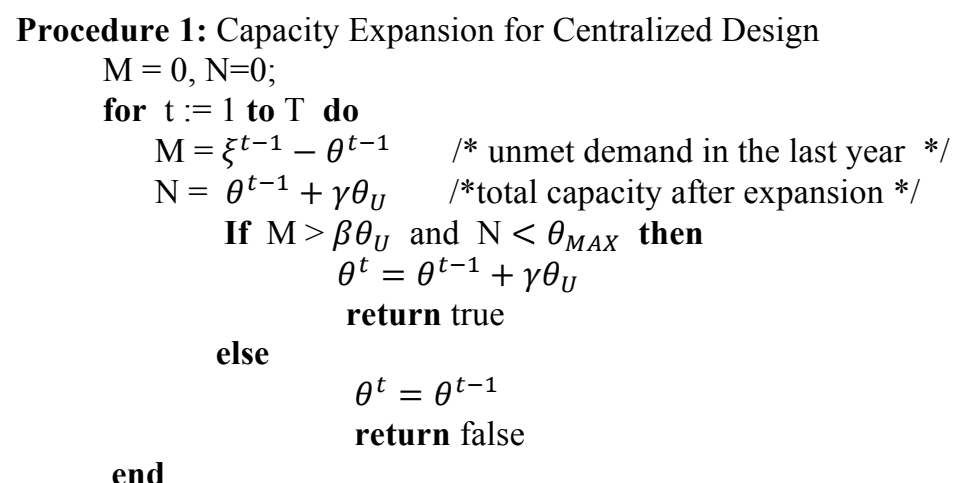


Variable $\theta_{U}$ is the unit capacity of a module (i.e., 50tpd in this study), $\beta$ is a decision variable, and $\beta \theta_{U}$ determines the threshold to expand the capacity. $\gamma$ is the number of modular designs that should be expanded. This determines the size of expansion.

According to the above decision rule, the capacity will be expanded when the amount of waste increases significantly. Costs that are related to capacity expansion should be considered in the evaluation process. The capacity planning model of the centralized design that considers both flexibility and uncertainty can be formulated as follows:

$$
\operatorname{Max} \quad E N P V=\sum_{s=1}^{2000} p_{s}\left(-\mathcal{C}^{0}\left(\theta_{s}^{0}\right)\left(1+C_{f}\right)+\sum_{t=1}^{T} \frac{A R_{t, s}\left(\theta_{s}^{t}, \xi_{s}^{t}\right)-A C_{t, s}\left(\theta_{s}^{t}, \xi_{s}^{t}\right)-C_{e x p}^{t}}{(1+\lambda)^{t}}\right)
$$

s.t. (10), (12)- (20), (25)-(26)

where $C_{\exp }^{t}$ is the capacity expansion cost in scenario s at year t. Note that the ENPV is maximized with respect to the initial capacity $\theta^{0}$, the capacity expansion threshold $\beta$ and the number of expanded modular designs $\gamma$.

\section{2) Flexible Decentralized Design}

The flexible decentralized design is similar to the flexible centralized design because it includes the option to add modular designs. The difference is that the decentralized design has the ability to install modular designs in all of the waste collection sectors, rather than just in the main site (i.e., Sector 6 in this case). Since the location problem is beyond the scope of the paper and the distances between each non-main sector (i.e., Sectors 1-5 in this case) are not known, it is challenging to select a nearby non-main sector with AD plants to dispose of the waste. To simplify the problem, it is assumed that the non-main sectors with $\mathrm{AD}$ plants only treat the waste within this sector, while the waste that cannot be treated in non-main sectors is transported and disposed at the main sector. 
Two additional decision rules have to be embedded to address the following questions: 1) should the modular designs be expanded in non-main sectors? 2) if they should be expanded in non-main sectors, which sector should be chosen for developing the new modular design?

The decision rule regarding the first question is: IF the capacity should be expanded AND IF the difference between the observed demand and the capacity at each waste collection sector (excluding the main site) reaches a threshold value THEN the modular designs are expanded in a decentralized manner:

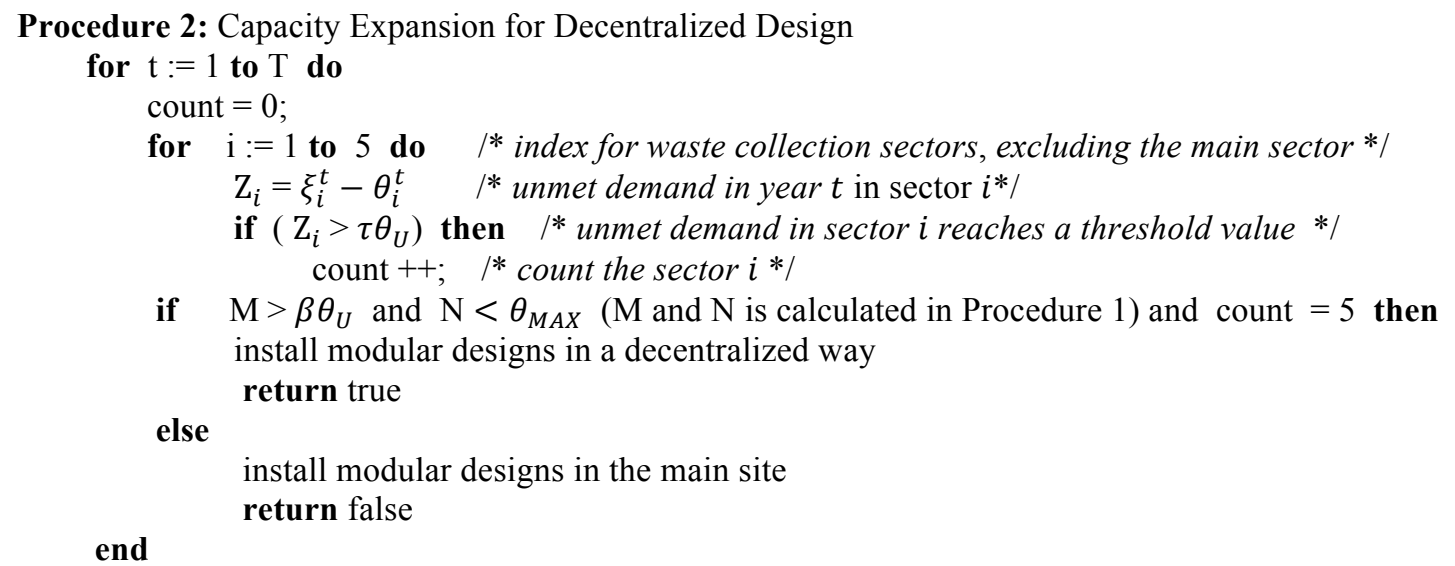

Here $\tau \theta_{U}$ determines the threshold value for expanding the modular designs in a decentralized way. The above procedure represents that $\mathrm{AD}$ modular designs can be developed in non-main sectors only when the waste is generated in a decentralized way (i.e., all the non-main sectors have unmet demands). In addition, these unmet demands in non-main sectors should be larger than a certain threshold. This may avoid situations whereby the capacity in non-main sectors is above the demand requirement (e.g., only treating the waste within the sector), while the capacity in the main sector is below the requirement (e.g., the main sector should treat all the waste that cannot be disposed of in non-main sectors). It should be noted that an AD modular design can be added in a decentralized way only when the whole system requires capacity expansion $\left(\mathrm{M}>\beta \theta_{U}\right.$ and $\mathrm{N}<\theta_{M A X}$ in Procedure 1$)$.

The decision rule regarding the second question is: IF the capacity should be expanded in a decentralized way (Procedure 2 is true) AND IF the transportation cost of sector $i(i=1,2, \cdots 5)$ that 
transports the unmet demand (i.e., demand that is larger than the current capacity) from sector $i$ to the main site (Sector 6) is larger than that of sectors $j(j \neq i, j=1,2, \cdots 5)$ THEN the modular designs are expanded in sector $i$.

Procedure 3: Select a Waste Collection Sector for Capacity Expansion

for $\mathrm{t}:=1$ to $\mathrm{T}$ do

$\operatorname{Max}=0 ; i \operatorname{Max}=0$;

for $i:=1$ to 5 do $\quad / *$ index for waste collection sectors, excluding the main sector */

$\operatorname{TranC}_{i}=\frac{\left(\xi_{i}^{t}-\theta_{i}^{t}\right)}{\operatorname{Cap}_{v}} * D_{\operatorname{Tr}_{i}} * C_{f u e l} ; / *$ costs for transporting the unmet demand to the main sector $* /$

if $\left(\operatorname{Max}<\operatorname{TranC}_{i}\right)$ then $\quad / *$ select a sector with maximum transportation costs */

$\operatorname{Max}=\operatorname{TranC}_{i}$

$i \operatorname{Max}=i$

if $\quad$ Procedure 2 is TRUE then

install modular designs in the sector with index $i$ Max

else

end

do nothing

$\xi_{i}^{t}-\theta_{i}^{t}$ represents the waste demand that cannot be treated in sector $i, \frac{\left(\xi_{i}^{t}-\theta_{i}^{t}\right)}{\operatorname{Cap}_{v}}$ represents the number of trips required to transport the unmet demand, and $\frac{\left(\xi_{i}^{t}-\theta_{i}^{t}\right)}{\operatorname{Cap}_{v}} * D_{\operatorname{Tr}_{i}} * C_{f u e l}$ represents the transportation costs of sector $i$. It should be noted that only one sector can be expanded per time period in this case. Based on Procedure 3, expanding the capacity in the sector with index iMax can save the largest transportation costs. The capacity expansion model of the decentralized design that considers both flexibility and uncertainty can be formulated as follows:

$\operatorname{Max} \quad E N P V=\sum_{s=1}^{2000} p_{s}\left(-\sum_{i=1}^{6} \mathcal{C}^{0}\left(\theta_{i s}^{0}\right)\left(1+C_{f}\right)+\sum_{t=1}^{T} \sum_{i=1}^{6} \frac{A R_{t, i, s}\left(\theta_{i s}^{t}, \xi_{i s}^{t}\right)-A C_{t, i, s}\left(\theta_{i s}^{t}, \xi_{i s}^{t}\right)-C_{e x p}^{t}}{(1+\lambda)^{t}}\right)$

$$
\text { s.t. (10), (12) - (16), (18) - (20), (22) - (23), (25) }
$$

It should be noted that the $E N P V$ here is maximized with respect to the initial capacity $\theta^{0}$, the capacity expansion threshold $\beta$, the number of expanded modular designs $\gamma$ and the decentralized threshold $\tau$.

\subsection{Results Discussion}

\subsubsection{Optimum Decision Rules}


To provide promising flexible designs to decision-makers, the initial design (i.e., the initial capacity) and the threshold to exercise flexibility (i.e., the decision variables $\beta, \gamma$ and $\tau$ ) should be first determined. In this study, a full factorial analysis was done to explore the design space with the goal of selecting the optimum thresholds using a simulation-based optimization approach [33, 34]. Since the design space was discretized, one should note that the solutions may not be global optima. The exhaustive enumeration technique was executed on a dedicated Dell Precision Tower T7910 workstation, equipped with a $2.4 \mathrm{GHz}$ Intel E5-2630 v3 processor and 32G of RAM. 2,000 scenarios for each optimization were implemented. The optimum thresholds were obtained within an acceptable time period due to computational speed of the model (i.e., 2 hours). TABLE 2 lists the feasible range for each variable and the incremental step sizes used in the enumeration. The optimum values for each variable under the conditions of EoS factor $\alpha=0.8$ and discount rate $\lambda=8 \%$ were analyzed. The results indicate that the best flexible centralized design installs an initial capacity of 200tpd, and then expands the capacity by 4 modules (i.e., $4 \times 50=200$ tpd) once the amount of waste collected in the previous year is larger than the current capacity by 50tpd (i.e., 1 modular design). The best flexible decentralized design is deployed by an additional decision rule that suggests expanding modular designs in other sectors if the food waste in each sector is larger than $50 \%$ of the modular capacity (i.e., $0.5 \times 50=25$ tpd). This decision rule can largely avoid unused capacity being deployed in each sector.

TABLE 2 DESIGN SPACE AND OPTIMUM DECISION RULES FOR FLEXIBILITY ANALYSIS

\begin{tabular}{cccc}
\hline \hline $\begin{array}{c}\text { Design variables and } \\
\text { decision variables }\end{array}$ & Values & $\begin{array}{c}\text { Increment } \\
\text { step size }\end{array}$ & $\begin{array}{c}\text { Optimum } \\
\text { value }\end{array}$ \\
\hline Initial capacity (tpd) & {$[200,600]$} & 100 & 200 \\
Capacity expansion threshold $\beta$ & {$[-3,3]$} & 1 & 1 \\
The number of expanded modular designs $\gamma$ & {$[1,5]$} & 1 & 4 \\
Decentralized threshold $\tau$ & {$[0,5]$} & 0.5 & 0.5 \\
\hline \hline
\end{tabular}

\subsubsection{Simulation Results}


The flexible centralized design and the flexible decentralized design are managed by the optimum decision rules discussed above. They are evaluated under the same 2,000 scenarios generated in the uncertainty analysis.

Fig. 3 compares the cumulative NPV distributions and ENPVs for the flexible centralized/decentralized designs and the benchmark design.

Fig. 3 NPVs of flexible centralized design and optimum fixed design

The results show that the flexible centralized design outperforms the benchmark design the overall effect of improving the system's ENPV. The ENPV of the flexible centralized S\$27 million, $S \$ 29$ million for the flexible decentralized, while the ENPV of the benchmark S\$22 million. This result supports the view that embedding flexibility in capacity expansion can improve lifecycle performance. It reverses the original design decision to build a largecentralized 600 tpd system mostly taking advantage from economies of scale. In addition, there chance - the value at gain (VAG) or percentile 95 value (P95) - that the NPV generated by the flexible centralized and decentralized designs will be greater than $\mathrm{S} \$ 32$ million and $\mathrm{S} \$ 34$ respectively, which is larger in both cases than that of the benchmark design ( $\$ \$ 30$ million). perspective of value at risk (VAR) - a measure of possible downside conditions - the design has a $5 \%$ chance of generating NPV less than $\mathrm{S} \$ 12$ million. This is much less than the of the flexible centralized and decentralized designs ( $\mathrm{S} \$ 21$ million and $\mathrm{S} \$ 23$ million indicating the latter designs are also good for mitigate exposure to downside risks.

Fig. 3 also shows the additional advantages of enabling flexibility to install modular designs in a decentralized way. It clearly shows that the flexible decentralized design can further improve the ENPV value compared to the flexible centralized design. This is because the flexible decentralized design not only reduces transportation costs, but also benefits from flexible modular deployment. 
TABLE 3 summarizes multiple comparison metrics. The best design is chosen based on the highest values for ENPV, P5 and P95 as well as the smallest value for standard deviation of NPV distributions.

TABLE 3 SUMMARY OF KEY STATISTICS FOR ALL DESIGNS (S\$ MILLION)

\begin{tabular}{ccccc}
\hline \hline & $\begin{array}{c}\text { Benchmark } \\
\text { design }\end{array}$ & $\begin{array}{c}\text { Flexible } \\
\text { centralized } \\
\text { design }\end{array}$ & $\begin{array}{c}\text { Flexible } \\
\text { decentralized } \\
\text { design }\end{array}$ & Best \\
\hline ENPV & 22 & 27 & 29 & Flexible Decentralized \\
P5 & 12 & 21 & 23 & Flexible Decentralized \\
P95 & 30 & 32 & 34 & Flexible Decentralized \\
Std.dev & 5.6 & 3.1 & 3.2 & Flexible \\
\hline \hline
\end{tabular}

A standard one-tail z-test (two samples for mean) was conducted to compare the ENPV of the flexible centralized and decentralized designs. The result of the z-test with $99 \%$ significance level yields $z=-32,050(p<0.01)$. Therefore, the sample data provide sufficient evidence to conclude that the ENPV of the flexible decentralized design is higher than that of the flexible centralized design. The result of the flexibility analysis provides a recommendation different from the deterministic and uncertainty analyses. It shows that the decentralized design becomes the best strategy when embedding flexibility. This result demonstrates that the benefits of flexibility can be greater than the benefits stemming from EoS in certain conditions. In addition, it shows that the value of a decentralized design is in the flexibility provided by the modularity rather than through the savings associated with reduced transportation costs.

\subsubsection{Sensitivity Analysis}

To illustrate the overall system performance, a two-way sensitivity analysis is conducted. The VOF of the flexible decentralized design is calculated (see Eq. 11) when the discount rate changes from $8 \%$ to $20 \%$ with a $2 \%$ increment and the EoS factor changes from 0.4 to 1.0 with a 0.1 step size. The results show that, under the same discount rate, the VOF increases as the EoS factor increases (i.e., economies of scale become weaker). This is because when EoS is weak, it is not worth building for large capacity 
all at once at time zero. Hence, the ENPV of the benchmark design decreases. In addition, there is more incentive to build small, and leverage the flexibility provided by the modularity when EoS becomes weaker, in line with Manne's [4] results. Also under the same EoS factor $\alpha=0.8$, the VOF increases when the discount rate increases. For example,

Fig. 4 clearly shows an increasing trend, due to the fact that a higher discount rate can largely reduce the present value of costs due to the deferral of investment. Therefore, the higher the discount rate $\lambda$, the more incentive there is to delay expansion and leverage flexibility, hence the higher VOF.

Fig. 4 Value of flexibility with different discount rates (EoS factor $\alpha=0.8)$

\section{Conclusion}

This study proposes a generic capacity planning model to generate the best strategies for deploying the capacity of flexible engineering systems under uncertainty. The form of the solution that is the output of the proposed model provides a systematic guideline for decision-makers managing flexibility for capacity expansion in operations. The capacity planning model builds upon existing methodologies, such as that developed by Manne [4], and enables investigating tradeoffs between EoS and the time value of money in the context of uncertainty and flexibility. This study is illustrated by selecting a capacity expansion strategy for WTE systems in Singapore. The results indicate that embedding flexibility can improve the economic performance of a system and outweigh, in certain conditions, the benefits from economies of scale. In addition, the decentralized design with flexibility outperforms all other designs under stated conditions. This is because the value of the flexible decentralized design is not only in reducing transportation costs, but also in the flexibility provided by the modularity, such as deferring expenses and avoiding unnecessary capacity deployment. The modeling approach and results can help designers, system architects, and decision-makers better recognize the value of flexibility in decentralized designs. It can also help with the evaluation of developing decentralized designs in small-scale cities, even though the savings from transportation 
costs may be low. This may alleviate concerns about over capacity supply, especially in an environment where economic growth is slowing down. A sensitivity analysis shows that strong EoS combined with a small discount rate encourages building more capacity now, while a weak EoS combined with a large discount rate encourages building small capacity now with flexible modular expansion in the future. The modeling framework can be used to fine tune and help decision-makers select the optimum capacity deployment strategies under different economic conditions.

Many opportunities for future research exist in addressing the limitations of this work. One of the limitations is that the results presented in this study are only valid from a lifecycle economic perspective. Future work may consider extending the model to multi-objective performance attributes (e.g., airborne emissions) to evaluate WTE systems from a sustainability perspective, complementary to the lifecycle economic approach presented here. In addition, only one source of uncertainty (i.e., recycled food waste) and one flexible strategy were analyzed in this study. A follow-up study (under way) could improve the case study by considering multiple sources of uncertainty (e.g., recycling rate, energy price, etc.) and multiple flexible strategies/real options (e.g., switching, abandoning, etc.) Other methodologies for modeling uncertainties can be considered, depending on the uncertainty driver(s) affecting the performance of the system of interest. Furthermore, it is not easy to solve the generic model when the size of the analytical problem is large. More work is needed to develop algorithmic methods that can efficiently solve the proposed model in more complex cases.

\section{Acknowledgments}

We are thankful for the financial support provided by the National Research Foundation Singapore under its Campus for Research Excellence and Technological Enterprise (CREATE), which is administered by the National University of Singapore and Shanghai Jiao Tong University. We are also grateful to Xie Qihui for his valuable inputs on the early economic model.

\section{References}


[1] de Neufville, R., and Scholtes, S., 2011, Flexibility In Engineering Design, MIT Press, Cambridge, MA, United States.

[2] de Neufville, R., 2008, "Lecture Notes," ESD.71: Engineering Systems Analysis for Design, Massachusetts Institute of Technology, Cambridge, MA, United States.

[3] K. Rapoza, 2014, "Real estate oversupply becoming bigger problem for China," Forbes.

[4] Manne, A. S., 1967, Investments for capacity expansion: size, location, and time-phasing, MIT Press.

[5] Ahmed, S., "Semiconductor tool planning via multi-stage stochastic programming," Proc. Proceedings of the International Conference on Modeling and Analysis in Semiconductor Manufacturing, pp. 153-157.

[6] Martínez-Costa, C., Mas-Machuca, M., Benedito, E., and Corominas, A., 2014, "A review of mathematical programming models for strategic capacity planning in manufacturing," International Journal of Production Economics, 153, pp. 66-85.

[7] Trigeorgis, L., 1996, Real options: Managerial flexibility and strategy in resource allocation, MIT Press.

[8] Cardin, M.-A., 2014, "Enabling flexibility in engineering systems: a taxonomy of procedures and a design framewrok," Journal of Mechanical Design, 136(1).

[9] de Neufville, R., Scholtes, S., and Wang, T., 2006, "Real options by spreadsheet: parking garage case example," Journal of infrastructure systems, 12, pp. 107-111.

[10] Cardin, M.-A., Ranjbar Bourani, M., and de Neufville, R., 2015, "Improving the Lifecycle Performance of Engineering Projects with Flexible Strategies: Example of On-Shore LNG Production Design," Systems Engineering, 18(3), p. 253.

[11] de Neufville, R., 2000, "Dynamic strategic planning for technology policy," International Journal of Technology Management, 19(3), pp. 225-245.

[12] Hreinsson, E. B., "Economies of scale and optimal selection of hydroelectric projects," Proc. Electric Utility Deregulation and Restructuring and Power Technologies, 2000. Proceedings. DRPT 2000. International Conference on, IEEE, pp. 284-289.

[13] Luss, H., 1982, "Operations research and capacity expansion problems: A survey," Operations Research, 30(5), pp. 907-947.

[14] Li, S., and Tirupati, D., 1994, "Dynamic capacity expansion problem with multiple products: Technology selection and timing of capacity additions," Operations Research, 42(5), pp. 958-976.

[15] Ryan, S. M., 2004, "Capacity expansion for random exponential demand growth with lead times," Management Science, 50(6), pp. 740-748. 
[16] Geng, N., Jiang, Z., and Chen, F., 2009, "Stochastic programming based capacity planning for semiconductor wafer fab with uncertain demand and capacity," European Journal of Operational Research, 198(3), pp. 899-908.

[17] Mittal, G., 2004, "Real options approach to capacity planning under uncertainty," Massachusetts Institute of Technology.

[18] de Neufville, R., Scholtes, S., and Wang, T., 2006, "Valuing Options by Spreadsheet: Parking Garage Case Example," ASCE Journal of Infrastructure Systems, 12(2), pp. 107-111.

[19] Dixit, A. K., 1994, Investment under uncertainty, Princeton University Press.

[20] National Environmental Agency, 2013, "Solid waste management," http://app2.nea.gov.sg/energy-waste/waste-management/refuse-disposal-facility.

[21] RIS international Ltd, 2005, "Feasibility of Generating Green Power through Anaerobic Digestion of Garden Refuse from the Sacramento Area," MacViro Consultants.

[22] Shell, 2014, "Shell Station Price Board," http://www.shell.com.sg/products-services/on-the$\mathrm{road} /$ fuels/price-board.html.

[23] National Environment Agency, 2011, "Environmental Protection Division Report ".

[24] IUT Global Pte Ltd, 2006, "9.5 MW food waste based grid connected power project implemented by IUT Singpaore Pte Ltd," Clean Development Mechanism.

[25] Singapore Power, 2013, "Electricity fariff rate," http://www.singaporepower.com.sg/irj/portal?NavigationTarget=navurl://41c8e6a3faf48bb168af2c222 faa8ee4\&windowId=WID1366188757420.

[26] Hu, J., and Cardin, M.-A., 2015, "Generating Flexibility in the Design of Engineering Systems to Enable Better Sustainability and Lifecycle Performance," Research in Engineering Design, 26(2), pp. $121-143$

[27] Bai, R., and Sutanto, M., 2002, "The practice and challenges of solid waste management in Singapore," Waste Management, 22(5), pp. 557-567.

[28] Evangelisti, S., Lettieri, P., Borello, D., and Clift, R., 2014, "Life cycle assessment of energy from waste via anaerobic digestion: A UK case study," Waste Management, 34(1), pp. 226-237.

[29] Ng, R. L. A. K., 2011, "Recycling firm IUT Global being wound up," The Business Times.

[30] Mikaelian, T., Rhodes, D. H., Nightingale, D. J., and Hastings, D. E., 2012, "A Logical Approach to Real Options Identification With Application to UAV Systems," IEEE Transactions on Systems, Man, and Cybernetics: Part A, 42(1), pp. 32-47

[31] Cardin, M.-A., Kolfschoten, G. L., Frey, D. D., de Neufville, R., de Weck, O. L., and Geltner, D. M., 2013, "Empirical Evaluation of Procedures to Generate Flexibility in Engineering Systems and Improve Lifecycle Performance," Research in Engineering Design, 24(3), pp. 277-295. 
[32] Lin, J., de Neufville, R., de Weck, O. L., and Yue, H. K. H., 2013, "Enhancing the Value of Oilfield Developments with Flexible Subsea Tiebacks," Journal of Petroleum Science and Engineering, 102, pp. 73-83.

[33] Martin, J. D., and Simpson, T. W., 2006, "A Methodology to Manage System-level Uncertainty During Conceptual Design," Journal of Mechanical Design, 128(4), pp. 959-968.

[34] Spall, J. C., 2003, Introduction to Stochastic Search and Optimization: Estimation, Simulation, and Control, Wiley.

\section{List of Table Captions}

TABLE 1 LIST OF ASSUMPTIONS FOR MODEL DEVELOPMENT

TABLE 2 DESIGN SPACE AND OPTIMUM DECISION RULES FOR FLEXIBILITY ANALYSIS

TABLE 3 SUMMARY OF KEY STATISTICS FOR ALL DESIGNS (S\$ MILLION)

\section{List of Figure Captions}

Fig. 1 NPV (expressed in S\$ million) of fixed designs capacities (expressed in tpd) under deterministic analysis

Fig. 2 (a) Recycled food waste in each sector; (b) GBM simulation of total recycled food waste (5 out of 2,000 runs)

Fig. 3 NPVs of flexible centralized design and optimum fixed design .......................................................24

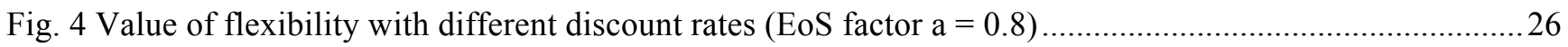

